Homopolymerisations

\begin{tabular}{|c|c|c|c|c|c|c|c|c|c|}
\hline ID & Cat. & $\mathrm{T} /{ }^{\circ} \mathrm{C}$ & $\begin{array}{c}p(E) / \\
\text { bar }\end{array}$ & $\begin{array}{c}\text { yield / } \\
\text { g }\end{array}$ & $t / \min$ & $\mathrm{n}$ (cat) / mol & $A \mathrm{I} / \mathrm{Zr}$ & $\begin{array}{c}\mathrm{V} \text { (solvent) / } \\
\mathrm{mL}\end{array}$ & c(1-hexene) / mol/L \\
\hline ACM14A & 2-DMS & 80 & 2.00 & 0.69 & 30 & 1.0E-07 & 17407 & 200 & 0 \\
\hline ACM14B & 2-DMS & 60 & 1.89 & 0.74 & 30 & $1.0 \mathrm{E}-07$ & 17407 & 200 & 0 \\
\hline ACM15A & 2-DMS & 40 & 1.38 & 0.92 & 30 & $1.0 \mathrm{E}-07$ & 17407 & 200 & 0 \\
\hline AHP6B & 2-DMS & 30 & 1.20 & 1.10 & 30 & $1.0 \mathrm{E}-07$ & 17407 & 200 & 0 \\
\hline ACM15B & 2-DMS & 10 & 1.07 & 1.20 & 30 & $1.0 \mathrm{E}-07$ & 17407 & 200 & 0 \\
\hline AHP21 & 2-TMS & 80 & 2.00 & 0.07 & 30 & 5.0E-07 & 3481 & 200 & 0 \\
\hline AHP20 & 2-TMS & 60 & 1.89 & 0.37 & 30 & $5.0 \mathrm{E}-07$ & 3481 & 200 & 0 \\
\hline AHP19B & 2-TMS & 40 & 1.38 & 0.69 & 30 & $5.0 \mathrm{E}-07$ & 3481 & 200 & 0 \\
\hline AHP19 & 2-TMS & 30 & 1.20 & 1.13 & 30 & $5.0 \mathrm{E}-07$ & 3481 & 200 & 0 \\
\hline AHP20B & 2-TMS & 10 & 1.07 & 0.96 & 30 & 5.0E-07 & 3481 & 200 & 0 \\
\hline
\end{tabular}

Copolymerisations

\begin{tabular}{|c|c|c|c|c|c|c|c|c|c|}
\hline ID & Cat. & $\mathrm{T} /{ }^{\circ} \mathrm{C}$ & $\begin{array}{c}p(E) / \\
\text { bar }\end{array}$ & $\begin{array}{c}\text { yield / } \\
\mathrm{g}\end{array}$ & $\mathrm{t} / \mathrm{min}$ & n(cat) / mol & $\mathrm{Al} / \mathrm{Zr}$ & $\begin{array}{c}\text { V(solvent) / } \\
\text { mL }\end{array}$ & c(1-hexene) / mol/L \\
\hline AHP13 & 2-DMS & 80 & 2.00 & 0.77 & 60 & 1.5E-07 & 11605 & 200 & 0 \\
\hline AHP7 & 2-DMS & 80 & 2.00 & 0.55 & 60 & $1.5 \mathrm{E}-07$ & 11605 & 200 & 0.036 \\
\hline AHP25 & 2-DMS & 80 & 2.00 & 0.59 & 60 & $2.0 \mathrm{E}-07$ & 8704 & 200 & 0.095 \\
\hline AHP9 & 2-DMS & 80 & 2.00 & 0.39 & 60 & 2.0E-07 & 8704 & 200 & 0.215 \\
\hline AHP25B & 2-DMS & 80 & 2.00 & 1.58 & 60 & 8.0E-07 & 2176 & 200 & 0.334 \\
\hline AHP9B & 2-DMS & 80 & 2.00 & 0.53 & 60 & 7.0E-07 & 2487 & 200 & 0.572 \\
\hline AHP26 & 2-DMS & 80 & 2.00 & 0.79 & 60 & $1.0 \mathrm{E}-06$ & 1741 & 200 & 0.698 \\
\hline AHP28B & 2-DMS & 80 & 0.79 & 0.56 & 60 & 1.0E-06 & 1741 & 200 & 0.381 \\
\hline AHP16B & 2-DMS & 80 & 0.58 & 0.95 & 60 & 2.0E-06 & 1741 & 200 & 0.382 \\
\hline AHP23 & 2-DMS & 80 & 0.27 & traces & 60 & 4.0E-06 & 1741 & 200 & 0.382 \\
\hline AHP27B & 2-DMS & 30 & 1.22 & 1.55 & 60 & $1.5 \mathrm{E}-07$ & 11605 & 200 & 0 \\
\hline AHP29 & 2-DMS & 30 & 1.22 & 0.81 & 60 & $1.5 \mathrm{E}-07$ & 11605 & 200 & 0.036 \\
\hline AHP29B & 2-DMS & 30 & 1.22 & 0.73 & 60 & 2.0E-07 & 8704 & 200 & 0.095 \\
\hline AHP30 & 2-DMS & 30 & 1.22 & 0.44 & 60 & 2.0E-07 & 8704 & 200 & 0.215 \\
\hline AHP30B & 2-DMS & 30 & 1.22 & 1.01 & 60 & 8.0E-07 & 2176 & 200 & 0.334 \\
\hline AHP31 & 2-DMS & 30 & 1.22 & 0.57 & 60 & 1.0E-06 & 1741 & 200 & 0.572 \\
\hline AHP31B & 2-DMS & 30 & 1.22 & 1.94 & 60 & $2.0 \mathrm{E}-06$ & 1741 & 200 & 0.698 \\
\hline AHP32 & 2-DMS & 30 & 0.932 & 1.62 & 60 & 2.0E-06 & 1741 & 200 & 0.721 \\
\hline AHP32B & 2-DMS & 30 & 0.685 & 1.81 & 60 & 2.0E-06 & 1741 & 200 & 0.721 \\
\hline AHP33 & 2-DMS & 30 & 0.317 & 3.68 & 60 & 4.0E-06 & 1741 & 200 & 0.708 \\
\hline
\end{tabular}




\section{PE 2-TMS}

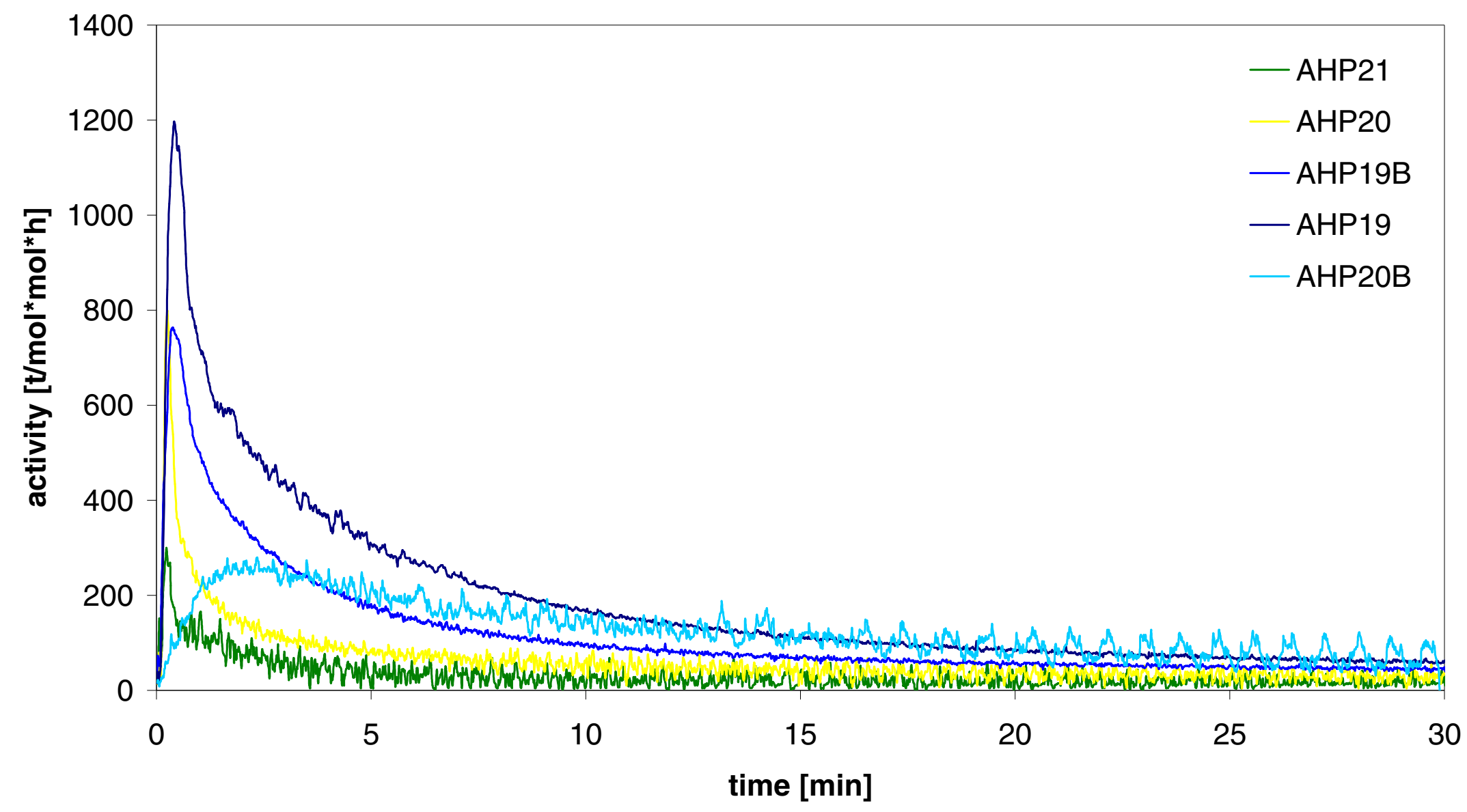


Copolymerisations (high activity)

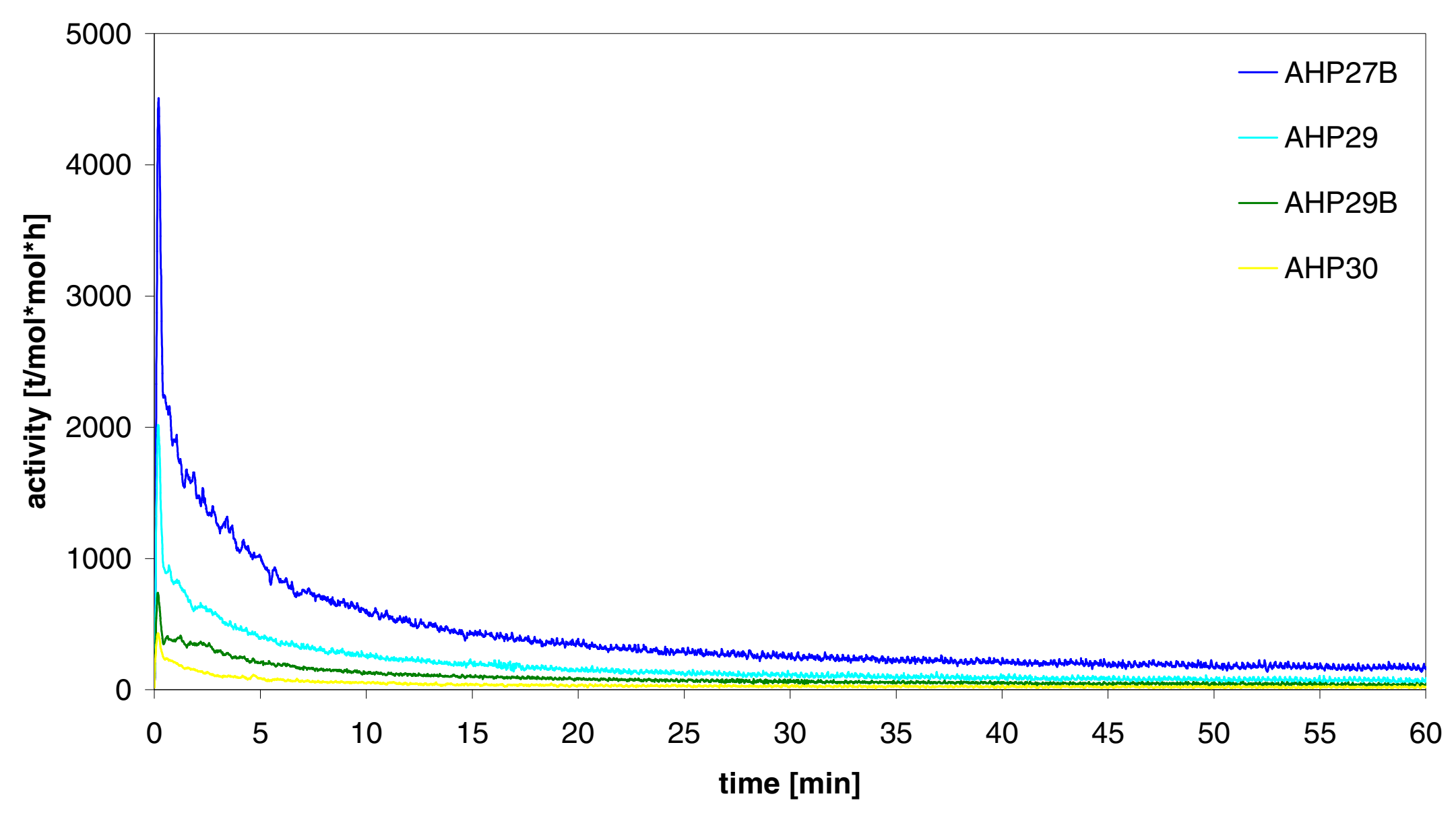

